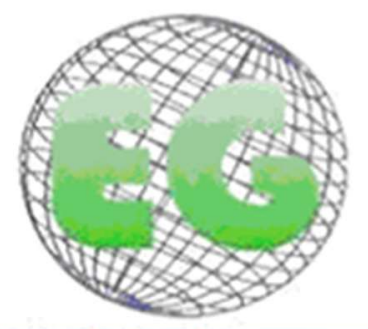

ISSN 1695-6141 $\mathbf{N}^{\circ} 63$

\title{
ORIGINALES
}

\section{Síntomas, comorbilidad y estado funcional de los pacientes con enfermedad renal crónica estadio 5 en manejo renal conservador} Symptom Burden, Comorbidity and Functional Status of patients with Chronic Kidney Disease Stage 5 managed conservately

\author{
Daniel Gutiérrez Sánchez ${ }^{1}$ \\ Juan P. Leiva-Santos²
}

1 Profesor Departamento de Enfermería y Podología. Universidad de Málaga. Málaga. España. danieltunie@hotmail.com

2 Médico responsable del Equipo de Soporte y Cuidados Paliativos Hospital de Manacor, Mallorca, Islas Baleares. Coordinador del Grupo de Estudio de Cuidados Paliativos Renales de la Sociedad Española de Nefrología (SEN). Miembro del Comité Técnico para la implementación de Cuidados Paliativos en Enfermedad Renal Crónica en Islas Baleares. Mallorca. España.

\section{https://doi.org/10.6018/eglobal.449531}

Recibido: 8/10/2020

Aceptado: 16/01/2021

\section{RESUMEN:}

Objetivos: Determinar la prevalencia e intensidad de síntomas pacientes con Enfermedad Renal Crónica estadio 5 en manejo renal conservador y analizar su asociación con la comorbilidad y el estado funcional.

Método: Estudio descriptivo, correlacional, de corte transversal. Para la evaluación de síntomas se utilizó la versión española modificada de la Palliative care Outcome Scale-Symptoms Renal. La comorbilidad fue evaluada con el índice de comorbilidad de Charlson modificado. Para la evaluación del estado funcional y grado de dependencia se utilizó el índice de Barthel. Resultados: 60 pacientes fueron incluidos en este estudio. Más del $50 \%$ de los pacientes presentaron debilidad, dolor, dificultad para dormir, poco apetito y problemas en la boca, encontrándose el dolor y la debilidad entre los síntomas más intensos. Las principales condiciones comórbidas fueron: diabetes, cardiopatía isquémica, enfermedad vascular periférica y neoplasias. La media del índice de Barthel fue $88 \pm 14,2$. No se encontró asociación entre la comorbilidad y la sintomatología $(p=0,43)$. El deterioro del estado funcional se asoció con la carga de síntomas $(p=0.001)$.

Conclusiones: Los pacientes con Enfermedad Renal Crónica estadio 5 en manejo renal conservador sufren una elevada carga de síntomas. La debilidad fue síntoma más frecuente en esta población. La carga sintomática se asoció con el deterioro del estado funcional. Futuros estudios sobre el impacto de los síntomas en estos pacientes, así como las intervenciones necesarias para su óptimo manejo deberían ser considerados.

Palabras clave: Enfermedad Renal Crónica Avanzada; Evaluación de síntomas; Medida de resultados; Cuidados paliativos 


\begin{abstract}
:
Objective: To determine the prevalence and severity of symptoms of patients with Chronic Kidney Disease Stage 5 managed conservately, and their association with the comorbidities and functional status.

Methods: A cross-sectional, descriptive, and correlational design was used. Symptom data were collected using the Spanish modified version of Palliative care Outcome Scale-Symptoms Renal. Comorbidity was collected and scored according to the modified Charlson Comorbidity Index. For the evaluation of functional status, the Barthel index was used.

Results: 60 patients were included in this study. More than $50 \%$ of patients described weakness, pain, difficulty for sleeping, poor appetite and mouth problems. Diabetes, coronary artery disease, and peripheral vascular disease, and tumor, were the main comorbid conditions of these patients. The mean score of Barthel index was $88 \pm 14.2$. There was no significant correlation between comorbidities and symptoms $(p=0.43)$. There was a significant correlation between symptoms and functional decline $(p=0.001)$.

Conclusions: Patients with Chronic Kidney Disease Stage 5 experience a high level of symptom burden. Weakness was the most prevalent symptom in this sample population. Symptom burden correlated with functional decline. Further longitudinal studies are needed that analyse the impact of symptoms and their management in this population.
\end{abstract}

Keywords: Advanced Chronic Kidney Disease; Symptoms assessment; Outcome measure; Palliative care

\title{
INTRODUCCIÓN
}

La Enfermedad Renal Crónica (ERC) es un problema cuya incidencia y prevalencia ha aumentado en los últimos años a nivel global(1). En España la prevalencia de Enfermedad Renal Crónica Avanzada (ERCA) (definida como ERC en estadio 4-5 con un filtrado glomerular $(F G)<30 \mathrm{ml} /$ min durante al menos tres meses) supera los 1.000 pacientes por millón de habitantes ${ }^{(2,3)}$.

Los pacientes con ERC estadio 5 sufren una amplia variedad de síntomas físicos y psicológicos con un elevado coste en atención y que además afectan negativamente a la calidad de vida(4-6). En este sentido, el personal de enfermería desempeña un papel esencial en la evaluación y manejo de la sintomatología del paciente en esta población ${ }^{(7)}$. Los síntomas no controlados en esta población contribuyen a un mayor sufrimiento y el manejo sintomático es una prioridad(8-11). En este contexto, la colaboración de los profesionales de las áreas de Nefrología y Cuidados Paliativos (CP) puede tener un efecto positivo en la calidad de vida de los pacientes y sus familias ${ }^{(10)}$. En este sentido, el paciente con ERC estadio 5 puede beneficiarse de los $\mathrm{CP}$, especialmente aquellos pacientes bajo manejo renal conservador (MRC) ${ }^{(11)}$. En esta línea, se considera que el MRC es una opción razonable para aquellos pacientes con comorbilidad elevada, edad avanzada y que no desean iniciar la diálisis ${ }^{(10,11)}$

Existe una escasez de estudios realizados en España sobre prevalencia de síntomas en pacientes en MRC, por lo que este aspecto debe ser investigado en mayor

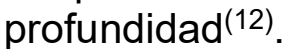

Los objetivos de este estudio fueron: (1) describir la prevalencia y la severidad de los síntomas en pacientes con ERC estadio 5 (FG <15 ml / min) en MRC, 2) explorar la asociación entre la sintomatología y la comorbilidad; y (3) explorar la asociación entre la sintomatología y el estado funcional. 


\section{MÉTODO}

\section{Participantes y procedimiento}

Estudio descriptivo, correlacional, de corte transversal. Todos los datos utilizados en este estudio se obtuvieron a partir de una amplia muestra de pacientes con ERCA como parte de un estudio más amplio en el que se realizó la adaptación transcultural y validación de un instrumento de medida de la sintomatología en esta población. En el presente estudio, se realizó un análisis descriptivo de la prevalencia y severidad de síntomas, la comorbilidad y el estado funcional de los pacientes con ERC estadio 5 en MRC. Además, se exploró la asociación de la sintomatología con la comorbilidad y el estado funcional.

Los pacientes fueron reclutados en el Servicio de Nefrología del Hospital Regional Universitario Carlos Haya de Málaga. Los datos del estudio fueron recogidos entre los meses de Abril y Septiembre de 2015.

Los criterios de inclusión fueron: (1) pacientes adultos de habla hispana con ERC estadio 5 (FG <15 ml / min) en MRC; y (2) consentimiento informado por escrito. Se excluyeron los pacientes con deterioro cognitivo y aquellos menores de 18 años.

Los pacientes fueron informados sobre el estudio y posteriormente se obtuvo un consentimiento informado por escrito. Se recogieron datos sociodemográficos y clínicos durante las entrevistas que tuvieron lugar en la consulta ERCA.

\section{Instrumentos}

\section{Versión española modificada de la Palliative care Outcome Scale-Symptoms Renal (POS-S Renal)}

La versión original de este instrumento fue diseñada para medir la sintomatología en pacientes con ERCA, y evalúa 17 síntomas $^{(13)}$. Esta versión original ha sido traducida y adaptada al español y ha demostrado ser un instrumento válido y fiable en la evaluación de la sintomatología en $\mathrm{ERCA}^{(14)}$. En este sentido, el cuestionario mostró unas propiedades psicométricas adecuadas en lo que a validez estructural, fiabilidad test-retest y validez de criterio se refiere ${ }^{(14)}$. Un nuevo ítem fue añadido a la versión española modificada del POS-S Renal (calambres). Como resultado, la versión española modificada de este cuestionario contiene 18 síntomas, y puede ser cumplimentada por el propio paciente ${ }^{(14)}$.

\section{Índice de comorbilidad de Charlson modificado (ICCm)}

Para evaluar la comorbilidad se utilizó el índice de comorbilidad de Charlson modificado $(\mathrm{ICCm})^{(15)}$. El ICCm es un índice de comorbilidad válido y fiable que ha sido ampliamente utilizado en población renal. El ICC contiene 19 condiciones comórbidas, que se puntúan de 1 a 6 , añadiéndose una puntuación de 1 por cada década a partir de los 40 años ${ }^{(15)}$. En esta versión modificada, el ítem "infarto agudo de miocardio" se ha sustituido por "enfermedad cardiovascular"(15). Se considera que una puntuación en el ICCm mayor o igual a 8 es altamente predictiva de mortalidad $^{(15)}$. 


\section{Índice de Barthel}

Para evaluar la capacidad funcional se utilizó el índice de Barthel(16). El índice de Barthel es un instrumento válido y fiable que ha sido ampliamente utilizado en población renal ${ }^{(17,18)}$. Este instrumento valora la independencia del paciente respecto a diez actividades básicas de la vida diaria: alimentación, aseo personal, vestirse, arreglarse, deposición, micción, uso de retrete, traslados, deambulación y subir escaleras $^{(16)}$. La puntuación de cada actividad es diferente, asignándose una puntuación de $0,5,10$ o 15 puntos. La puntuación total oscila de 0 (dependencia total) a 100 puntos (independencia) ${ }^{(16)}$.

\section{Análisis estadístico}

Se realizó un análisis descriptivo de las variables sociodemográficas y clínicas de la población. Se utilizaron estadísticos de tendencia central (media \pm desviación estándar) para las variables continuas y porcentajes para las variables categóricas.

Para describir la sintomatología se utilizaron porcentajes e intervalos de confianza (IC) al 95\%. Se realizó la prueba de Kolmogorov-Smirnov para determinar la distribución de la muestra. Para explorar la relación de la sintomatología con la comorbilidad y el estado funcional del paciente se utilizó el coeficiente de correlación de Pearson. Se tomó el valor de $p<0,05$ como estadísticamente significativo. Los análisis se realizaron utilizando el software de análisis estadístico SPSS versión 24.

\section{Consideraciones éticas}

Este estudio ha sido aprobado por el Comité de Ética Provincial de Málaga. Los estándares de buena práctica clínica y los principios éticos establecidos para la investigación en seres humanos, de acuerdo con la Declaración de Helsinki y sus revisiones posteriores, se mantuvieron en todo momento.

Los datos clínicos se separaron de los datos de identificación personal y las bases de datos se cifraron y almacenaron en dispositivos específicamente reservadas para este proyecto. Previa participación en el estudio, los cuidadores recibieron información sobre el contenido y el alcance del estudio y dieron su consentimiento por escrito.

\section{RESULTADOS}

\section{Características de la muestra}

60 pacientes con ERC estadio 5 en MRC fueron incluidos en este estudio. Las características sociodemográficas y clínicas se muestran en la Tabla 1. Los valores perdidos fueron menores al $2 \%$. La media del ICCm fue $6,2 \pm 2,2$ y las principales condiciones comórbidas fueron: diabetes, cardiopatía isquémica, neoplasias y enfermedad vascular periférica (Tabla 2). La media del índice de Barthel fue $88 \pm 14,2$ (Tabla 3). 
Tabla 1.Características sociodemográficas y clínicas $(\mathrm{N}=60)$

\begin{tabular}{lcc}
\hline Características & $\mathbf{n}$ & $\mathbf{\%}$ \\
\hline Edad (media, DE) & 70,60 & $\pm 9,2$ \\
Género & 39 & 65 \\
Hombre & 21 & 35 \\
Mujer & 21,3 & $\pm 2,3$ \\
Filtrado glomerular (ml/min/1,73 m2) (media, DE) & 25,7 & $\pm 20,2$ \\
Tiempo en MRC (meses) & & \\
Causas de la ERC & 27 & 45 \\
Enfermedad vascular & 9 & 15 \\
Nefropatía diabética & 6 & 10 \\
Enfermedad poliquística & 4 & 6,7 \\
Enfermedad glomerular & 10 & 16,7 \\
Etiología desconocida & 4 & 6.7 \\
Otras causas & \multicolumn{2}{c}{} \\
\hline
\end{tabular}

Fuente: Datos de la investigación

Las cantidades representan frecuencia $(n)$, porcentajes (\%) o media (DE)

Tabla 2. ICCm y prevalencia de condiciones comórbidas $(\mathrm{N}=60)$

\begin{tabular}{lcc}
\hline Condiciones comórbidas & $\mathbf{n}$ & $\mathbf{\%}$ \\
\hline Cardiopatía isquémica & 13 & 21,7 \\
Insuficiencia cardíaca congestiva & 11 & 18,3 \\
Enfermedad vascular periférica & 6 & 10 \\
Enfermedad cerebrovascular & 3 & 5 \\
Demencia & 0 & 0 \\
EPOC & 3 & 5 \\
Enfermedad del tejido conectivo & 4 & 6,7 \\
Enfermedad ulcerosa & 3 & 5 \\
Enfermedad hepática leve & 2 & 3,3 \\
Diabetes mellitus & 9 & 15 \\
Hemiplejia & 0 & 0 \\
Enfermedad renal moderada o severa & 60 & 100 \\
Diabetes con daño orgánico & 18 & 30 \\
Tumor localizado, leucemia, linfoma o mieloma multiple & 12 & 20 \\
Enfermedad hepática moderada o severa & 1 & 1,7 \\
Tumor sólido secundario metástasico & 0 & 0 \\
SIDA & 0 & 0 \\
ICC (media, DE) & 6,2 & $\pm 2,2$ \\
\hline \multicolumn{2}{c}{ Fuente: Datos de la investigación }
\end{tabular}

Fuente: Datos de la investigación

Las cantidades representan frecuencia (n), porcentajes (\%) o media (DE) 
Tabla 3. Índice de Barthel y grado de deterioro functional $(\mathrm{N}=60)$

\begin{tabular}{lcc}
\hline Nivel de dependencia & $\mathbf{n}$ & $\%$ \\
\hline Dependencia total (Barthel 0-15) & 0 & 0 \\
Dependencia grave (Barthel 20-35) & 1 & 1,7 \\
Dependencia moderada (Barthel 40-55) & 2 & 3,4 \\
Dependencia no relevante (Barthel 60-100) & 57 & 94,9 \\
Indice de Barthel (media, DE) & 88 & $\pm 14,2$ \\
\hline
\end{tabular}

Fuente: Datos de la investigación

Las cantidades representan frecuencia (n), porcentajes (\%) o media (DE)

\section{Prevalencia y severidad de los síntomas}

Los síntomas más frecuentes fueron: debilidad $-81,4 \%$ (IC: $71-92 \%$ ), dolor $-58,3 \%$ (IC: $42-69 \%$ ), dificultad para dormir - 58,3\% (IC: $48-75 \%$ ), poco apetito - 55\% (IC: $40-$ $67 \%$ ), problemas en la boca $-53,3 \%$ (IC: $35-60 \%$ ), depresión - 45\% (Cl: 33-60\%), cambios en la piel - 45\% (IC: $29-56 \%$ ), estreñimiento $36,7 \%$ (IC: $24-50 \%$ ), poca movilidad - 36,2\% (IC: $24-50 \%$ ), ansiedad - 35\% (IC: $24-50 \%$ ), disnea-33,9\% (IC: $20-$ $46 \%$ ), somnolencia - 33,3\% (IC: $24-50 \%$ ), prurito - 32,2\% (IC: $22-48 \%$ ), calambres$32 \%$ (IC: $20-46 \%$ ) y náuseas-31,7\% (IC: 19-44\%) . La prevalencia de síntomas se muestra en la figura 1.

El número medio de síntomas padecidos por paciente fue de 6,7 $\pm 3,6$ de un máximo de 18, con un rango de 0-15 síntomas por paciente. Los síntomas más intensos (de severo a insoportable) fueron: el dolor, la debilidad, el estreñimiento y el prurito (Figura 2).

Figura 1. Prevalencia de síntomas

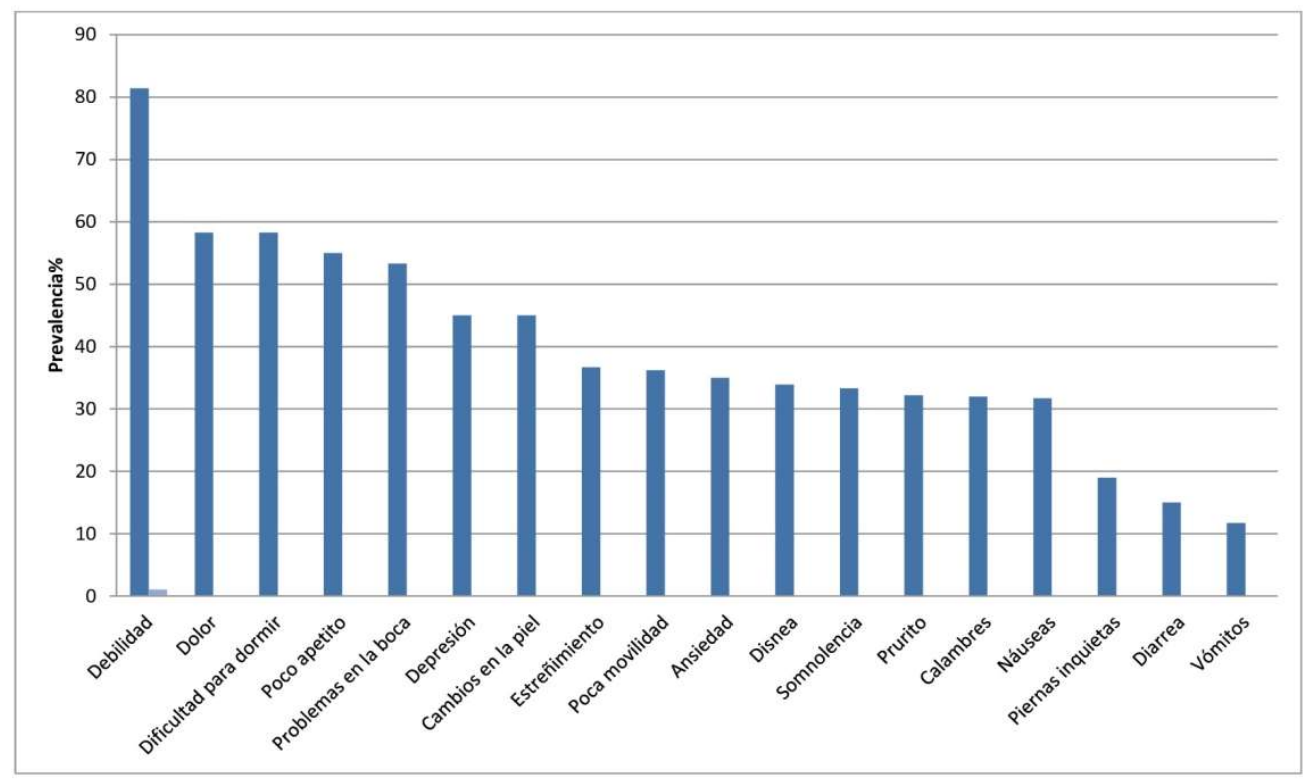

Fuente: Datos de la investigación. Las cantidades representan porcentajes (\%) 
Figura 2. Severidad de síntomas

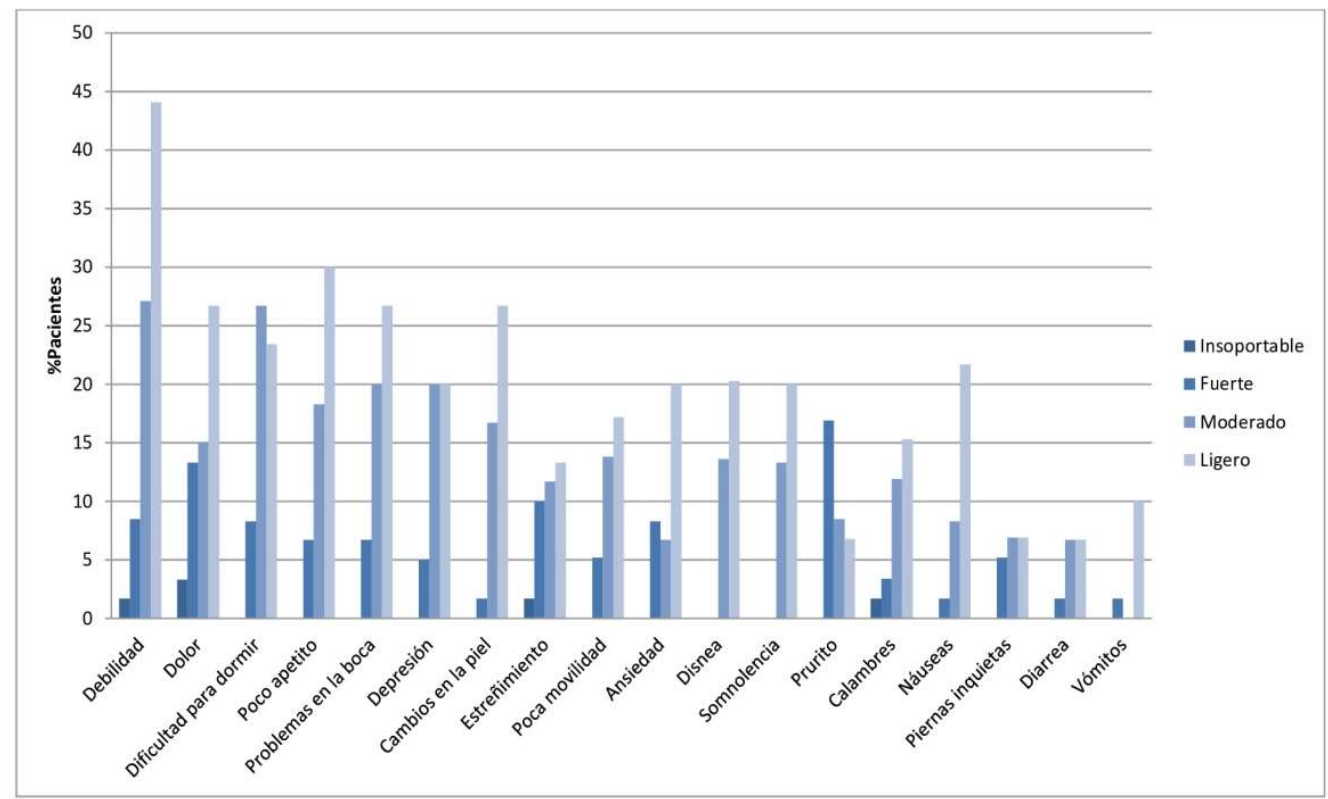

Fuente: Datos de la investigación. Las cantidades representan porcentajes (\%)

\section{Asociación entre los síntomas y la comorbilidad}

Se evaluó la asociación entre la sintomatología y la comorbilidad. Los resultados indicaron que no hubo correlación significativa entre la carga sintomática y la comorbilidad $(r=0,11, p=0,43)$.

\section{Asociación entre los síntomas y la funcionalidad}

También se evaluó la asociación entre los síntomas y el estado funcional del paciente. Los resultados obtenidos indicaron que existía una asociación negativa entre la carga de síntomas y el estado funcional $(r=-0,48, p=0,001)$, y por tanto la carga de síntomas fue mayor en aquellos pacientes con peor estado funcional.

\section{DISCUSIÓN}

La sintomatología en pacientes con ERC estadio 5 en MRC está considerada como un constructo difícil de medir, ya que está determinado por aspectos físicos y psicológicos, y está influenciada por factores como la propia insuficiencia renal, la comorbilidad o el tratamiento(5,19,20). Además, los síntomas en esta población no se presentan de manera aislada, sino más bien en forma de grupos de síntomas ${ }^{(21)}$. En este sentido, la realización de estudios que aborden la sintomatología en esta población permite optimizar el manejo sintomático.

Hasta donde sabemos, este es el primer estudio que se realiza en España que analiza la prevalencia y severidad de síntomas en ERC estadio 5 en pacientes en MRC y su asociación con la comorbilidad y el estado funcional.

Los resultados de este estudio indican que los pacientes con ERC estadio 5 en MRC presentan una elevada carga de síntomas y comorbilidad, aunque mantienen un 
estado funcional aceptable. Síntomas como la debilidad $(81,4 \%)$, y el dolor $(58,3 \%)$ presentaron una frecuencia elevada en estos pacientes. Además, más del $50 \%$ de los pacientes presentaron debilidad, dolor, dificultad para dormir, poco apetito y problemas en la boca, encontrándose el dolor y la debilidad entre los síntomas más intensos. Estos síntomas son consistentes en la mayoría de estudios y se asocian con una peor calidad de vida(5,6,22).

La debilidad continúa siendo un síntoma muy frecuente en esta población y se asocia con otros síntomas como la depresión y los trastornos del sueño, y además tiene un impacto en el estado funcional del paciente, lo que sugiere que un manejo adecuado de la carga sintomática global con un enfoque multidimensional podría disminuir la frecuencia e intensidad de este síntoma (23). En este estudio la debilidad fue el síntoma más frecuente e intenso. Estos hallazgos fueron comparables a los descritos en otros estudios ${ }^{(5,19,23)}$.

El dolor continúa siendo un síntoma prevalente en esta población. La etiología del dolor en esta población suele estar relacionada con los problemas vascularesisquémicos, la neuropatía periférica, y los problemas musculares y osteoarticulares ${ }^{(24)}$. Además el dolor también está relacionado con otros síntomas como el insomnio y la depresión, y también tiene un efecto en el estado funcional del paciente ${ }^{(25)}$. En este estudio el dolor fue el segundo síntoma más frecuente e intenso. Estos resultados son comparables a los descritos en otros estudios ${ }^{(5,19)}$.

En cuanto a la asociación entre la carga sintomática y la comorbilidad, no se encontró correlación significativa $(p=0,43)$. Estos hallazgos fueron comparables a los descritos en otros estudios ${ }^{(21)}$.

Respecto a la asociación entre la carga sintomática y la capacidad funcional, se encontró una asociación negativa y significativa $(p=0,001)$, lo que indica que la carga de síntomas fue mayor en aquellos pacientes con peor estado funcional. En este sentido, la carga sintomática tiene un efecto negativo sobre la capacidad funcional y el grado de dependencia de esta población ${ }^{(26)}$.

La atención paliativa en esta población puede tener un impacto positivo optimizándola el manejo sintomático de estos pacientes ${ }^{(27,28)}$. Por lo tanto, los principios de los $\mathrm{CP}$ deberían integrarse en la atención de los pacientes con ERCA, especialmente en pacientes en MRC(10,11). Esto es especialmente importante en España, donde la integración de los principios de los CP en los Servicios de Nefrología está en desarrollo.

Este estudio muestra el valor de la evaluación y monitorización sintomática a través de instrumentos validos y fiables como la versión española modificada del POS-S Renal como un instrumento que facilita al clínico una óptima evaluación de los síntomas en $\mathrm{ERCA}^{(14)}$. En este sentido, el personal de enfermería desempeña un papel fundamental en la evaluación y manejo de la sintomatología del paciente en $\operatorname{MRC}^{(7)}$.

Este estudio tiene varias limitaciones. Se trata de un estudio transversal que se ha realizado en un solo centro y por tanto los resultados no son directamente transferibles a otros centros. En este sentido, se necesitan estudios multicéntricos adicionales que usen una población más amplia. 


\section{CONCLUSIONES}

Los pacientes con ERC estadio 5 en MRC sufren una elevada carga de síntomas que condiciona negativamente el estado funcional del paciente. La debilidad fue síntoma más frecuente en esta población. Futuros estudios sobre el impacto de los síntomas en estos pacientes, así como las intervenciones necesarias para su óptimo manejo deberían ser considerados.

\section{REFERENCIAS}

1. Bikbov B, Purcell CA, Levey AS, Smith M, Abdoli A, Abebe M, et al. Global, regional, and national burden of chronic kidney disease, 1990-2017: a systematic analysis for the Global Burden of Disease Study 2017. The Lancet. 2020 Feb 29;395(10225):709-33. Disponible en: https://doi.org/10.1016/S0140-6736(20)300453

2. Gorostidi M, Sánchez-Martínez M, Ruilope LM, Graciani A, de la Cruz JJ, Santamaría R, et al. Chronic kidney disease in Spain: Prevalence and impact of accumulation of cardiovascular risk factors. Nefrología (English Edition). $2018 \mathrm{Nov}$ 1;38(6):606-15. Disponible en: https://doi.org/10.1016/j.nefroe.2018.04.010

3. K/DOQI Clinical practice guidelines for chronic kidney disease: evaluation, classification, and stratification. Am J KidneyDis. 2002;39(2 Suppl1):1-266.

4. Davison SN, Levin A, Moss AH, Jha V, Brown EA, Brennan F, et al. Executive summary of the KDIGO Controversies Conference on Supportive Care in Chronic Kidney Disease: developing a roadmap to improving quality care. Kidney international. 2015;88(3):447-59.1. doi:10.1038/ki.2015.110

5. Murtagh FE, Addington-Hall J, Edmonds P, Donohoe P, Carey I, Jenkins K, et al. Symptoms in the month before death for stage 5 chronic kidney disease patients managed without dialysis. J Pain Symptom Manage. 2010 Sep;40(3):342-52. Disponible en: https://doi.org/10.1016/j.jpainsymman.2010.01.021

6. Abdel-Kader K, Unruh ML, Weisbord SD. Symptom burden, depression, and quality of life in chronic and end-stage kidney disease. Clinical journal of the American Society of Nephrology : CJASN. 2009;4(6):1057-64. Disponible en: https://doi.org/10.2215/CJN.00430109

7. Barrett BJ, Garg AX, Goeree R, Levin A, Molzahn A, Rigatto C, et al. A Nursecoordinated Model of Care versus Usual Care for Stage 3/4 Chronic Kidney Disease in the Community: A Randomized Controlled Trial. Clin J Am Soc Nephrol. 2011 Jun;6(6):1241-7. Disponible en: https://doi.org/10.2215/CJN.07160810

8. Steinhauser KE, Christakis NA, Clipp EC, McNeilly M, Mclntyre L, Tulsky JA. Factors considered important at the end of life by patients, family, physicians, and other care providers. JAMA. 2000;284(19):2476-82.

9. Manns B, Hemmelgarn B, Lillie E et al. Setting research priorities for patients on or nearing dialysis. Clin J Am Soc Nephrol 2014; 9: 1813-1821. Disponible en: https://doi.org/10.2215/CJN.01610214

10. Leiva-Santos JP, Sánchez-Hernández R, García-Llana H, Fernández-Reyes MJ, Heras-Benito M, Molina-Ordas Á, et al. Cuidados de soporte renal y cuidados paliativos renales: revisión y propuesta en terapia renal sustitutiva. Nefrología (Madrid). 2012;32(1):20-7. Disponible en: http://scielo.isciii.es/scielo.php?script=sci abstract\&pid=S021169952012000100005\&Ing=es\&nrm=iso\&tIng=es 
11. Brown MA, Crail SM, Masterson R, Foote C, Robins J, Katz I, et al. ANZSN renal supportive care 2013: opinion pieces [corrected]. Nephrology (Carlton). 2013 Jun;18(6):401-54. Disponible en:https://doi.org/10.1111/nep.12065

12. Gutiérrez Sánchez D, Leiva-Santos JP, Sánchez-Hernández R, Gómez García R. Prevalencia y evaluación de síntomas en enfermedad renal crónica avanzada. Enfermería Nefrológica. 2015 Sep;18(3):228-36. Disponible en: https://doi.org/10.4321/S2254-28842015000300010

13. Murphy EL, Murtagh FEM, Carey I, Sheerin NS. Understanding symptoms in patients with advanced chronic kidney disease managed without dialysis: use of a short patient-completed assessment tool. Nephron Clinical Practice. 2009;111(1):7480. Disponible en: https://doi.org/10.1159/000183177

14. Gutiérrez-Sánchez D, Leiva-Santos JP, Sánchez-Hernández R, HernándezMarrero D, Cuesta-Vargas Al. Spanish modified version of the palliative care outcome scale-symptoms renal: cross-cultural adaptation and validation. BMC Nephrol. 2016 Nov 18;17(1):180. Disponible en: https://doi.org/10.1186/s12882-016-0402-8

15. Beddhu S, Bruns FJ, Saul M, et al. A simple comorbidity scale predicts clinical outcomes and costs in dialysis patients. Am JMed 2000;108(8):609-13. Disponible en: https://doi.org/10.1016/S0002-9343(00)00371-5

16. Mahoney FI, Barthel DW. FUNCTIONAL EVALUATION: THE BARTHEL INDEX. Maryland State Medical Journal. 1965 Feb;14:61-5.

17. Andreu Periz L, Andreu-Periz L, Puig Llobet M, Puig-Llobet M, Cases Amenós A, Cases-Amenós A. Grado de dependencia de las personas sometidas a hemodiálisis en Cataluña y evolución de su mortalidad. Nefrología. 2012 Sep 1;32(5):613-21. Disponible en: https://doi.org/10.3265/Nefrologia.pre2012.Jun.11460

18. Cook WL, Jassal SV. Functional dependencies among the elderly on hemodialysis. Kidney International. 2008 Jun 1;73(11):1289-95. Disponible en: https://doi.org/10.1038/ki.2008.62

19. Murtagh FEM, Addington-Hall JM, Edmonds PM, Donohoe P, Carey I, Jenkins K, et al. Symptoms in advanced renal disease: a cross-sectional survey of symptom prevalence in stage 5 chronic kidney disease managed without dialysis. J Palliat Med. 2007 Dec;10(6):1266-76. Disponible en: https://doi.org/10.1089/jpm.2007.0017

20. Gutiérrez Sánchez D, Leiva-Santos JP, López M, José M, Vargas C, I A. Perfil sintomático de los pacientes con Enfermedad Renal Crónica Estadio 4 y 5 . Enfermería Nefrológica. 2017 Sep;20(3):259-66. Disponible en: https://doi.org/10.4321/s225428842017000300010

21. Gutiérrez Sánchez D, Leiva-Santos JP, Cuesta-Vargas Al. Symptom Burden Clustering in Chronic Kidney Disease Stage 5. Clin Nurs Res. 2017 Nov 1;1054773817740671. Disponible en: https://doi.org/10.1177/10547738177440671

22. Murtagh FEM, Addington-Hall J, Higginson IJ. The prevalence of symptoms in end-stage renal disease: a systematic review. ACKD2007;14(1):82-99. Disponible en: https://doi.org/10.1053/i.ackd.2006.10.001

23. Almutary $\mathrm{H}$, Douglas $\mathrm{C}$, Bonner A. Multidimensional symptom clusters: an exploratory factor analysis in advanced chronic kidney disease. J Adv Nurs. 2016 Oct;72(10):2389-400. Disponible en: https://doi.org/10.1053/i.ackd.2006.10.001

24. Davison SN, Koncicki H, Brennan F. Pain in chronic kidney disease: a scoping review. Semin Dial. 2014 Mar;27(2):188-204. Disponible en: https://doi.org/10.1111/sdi.12196

25. Davison SN, Jhangri GS. Impact of pain and symptom burden on the healthrelated quality of life of hemodialysis patients. JPSM. 2010 Mar;39(3):477-85. Disponible en: https://doi.org/10.1016/j.jpainsymman.2009.08.008 
26. Weiner DE, Seliger SL. Cognitive and Physical Function in Chronic Kidney Disease. Curr Opin Nephrol Hypertens. 2014 May;23(3):291-7. Disponible en: https://doi.org/10.1097/01.mnh.0000444821.87873.7b

27. Tamura MK, Meier DE. Five policies to promote palliative care for patients with ESRD. Clin J Am Soc Nephrol 2013; 8: 1783-1790. Disponible en: https://doi.org/10.2215/CJN.02180213

28. Kurella Tamura M, O'Hare AM, Lin E, Holdsworth LM, Malcolm E, Moss AH. Palliative Care Disincentives in CKD: Changing Policy to Improve CKD Care. American Journal of Kidney Diseases: The Official Journal of the National Kidney Foundation. 2018;71(6):866-73. https://doi.org/10.1053/j.ajkd.2017.12.017

Disponible en: 